\title{
Model Predictive Control for Positioning and Navigation of Mobile Robot with Cooperation of UAV
}

\author{
Moustafa M. Kurdi \\ Belarusian National Technical \\ University \\ Minsk, Belarus
}

\author{
Imad A. Elzein \\ Belarusian National Technical \\ University \\ Minsk, Belarus
}

\author{
Alex K. Dadykin \\ Belarusian National Technical \\ University \\ Minsk, Belarus
}

\begin{abstract}
The purpose of navigation system is to help mobile robot in order to select an optimal and short path to reach the target. In most of these systems, GPS are used to determine the robot position. There are errors in positioning using GPS. This paper considers the problem of navigating a Mobile Robot in an unknown environment while maintaining visibility with a (movable or non-movable) target by means of Fuzzy Model Predictive Control (FMPC). The approach combines input variables from different resources such as: GPS, RVS (Robot Vision System), and QVS (Quad-copter Vision System). In this paper, a new approach based on Fuzzy Model Predictive Control (FMPC) is proposed to solve the positioning and navigation problems for mobile robot.
\end{abstract}

\section{General Terms}

Navigation; Model Predictive Control; Mobile Robot

\section{Keywords}

Navigation; Fuzzy; MPC; Mobile Robot; UAV

\section{INTRODUCTION}

Mobile robotics is the area of robotics that groups the robots that have no fixed base, or they can get around within a limited or no space. Robot's locomotion should be recognized as a moving object, save its position within this space understand the physical limits of space and adapt providing ways and fixed objects that can be obstacles to be diverted.

The robots have potential application in areas such as (i) Support to medical services (Transportation of food, medication, medical exams); (ii) Automatic cleaning of areas (Supermarkets, airports, industrial sites); (iii) Agricultural and Forests (fertilization, planting, fire preventing, tree cutting); (iv) Hazard Environments (catastrophic areas, volcanoes, nuclear power plants, inspection of gas or oil pipes); (v) Military (Surveillance vehicles, Monitoring vehicles); (vi) Safety ( Surveillance of large areas, buildings, airports, car parking); and (vii) Civil Transportation (Inspection of airplanes, trains).

A 4-wheel mobile robot is a robot that can move in any direction in the horizontal plane depending on angle of rotation $\Theta$. Thus, for locomotion, it is necessary that it has a computer embedded system responsible for controlling all its functions. This computer system can send and receive information so that the robot can perform its functions. Thus, this is called a control system. This system can be divided into simpler control modules.

The Mobile Robot is a strongly coupled system which influence the rotation of front wheels directly influences the speed of the other two wheels, as well as the resulting velocity of the center of mass of the robot. For this coupled system, nonlinear and MIMO (Multiple Inputs Multiple outputs) developed a multivariable control.

A major difficulty in a mobile robot design is the controller design for trajectory tracking. The trajectory control may be accomplished through numerous control techniques. When considering a path that faithfully represents the desired trajectory, it is necessary to introduce an approach that incorporates a data feed related to the actual location of the mobile robot. This perspective is fundamental to running a control to ensure the displacement of the robot in a desired trajectory.

The main objective of this work is to introduce and develop a multivariable controller for mapping 4-wheel mobile robot path using a model in state space that allows the robots to move smoothly with obstacles avoidance. Robot's position plays a key role in path tracking. The objective of the study of path planning in mobile robotics is to give robots the ability to plan their own movements, without the need for direct interference of humans. As a result, our approach is to use model predictive control for robot trajectory generation (navigation) and positioning.

Path trajectory for a four-wheel mobile robot (Belarus 132N) is in fact a feedback control problem to which model-based predictive control (MPC) has been an effective mechanism $[1,2]$. Most MPC control applications are based on linear models of dynamic systems to predict outputs over a certain horizon. Future sequences of control signals are evaluated by minimizing a cost index that takes account of the future output prediction errors over a reference trajectory, as well as control efforts. However, when the systems are non-linear and are operated over a large dynamic range, the use of linear models becomes impractical, and the identification of non-linear models for control becomes absolutely necessary.

Fuzzy Model Predictive Control (FMPC) [3], [4] combines input variables from different resources such as: GPS, robot image system, and quad-copter video system in order to achieve localization i.e. Cartesian coordinates $(x, y)$ and the orientation $\theta$

This paper is organized as follows: Section II presents the four wheeled mobile robot design, and Section III presents the fuzzy model predictive control. The proposed control system is demonstrated in Section IV. Our conclusions and thoughts on future extensions are summarized in Section V.

\section{FOUR WHEELED MOBILE ROBOT DESIGN}

The wheel has been by far the most popular locomotion (how the robot moves through its environment) mechanism in mobile robotics and in man-made vehicles in general. It can 
achieve very good efficiencies, and it does so with a relatively simple mechanical implementation. Almost all wheeled robot research tends to focus on the problems of traction and stability, maneuverability, and control: can the robot wheels provide sufficient traction and stability for the robot to cover all of the desired terrain, and does the robot's wheeled configuration enable sufficient control over the velocity of the robot?

\subsection{Design of Belarus-132N Mobile Robot}

The Mobile robot used in the experiment use chassis tractors "Belarus-132N" platform with the petrol engine (HONDA GX390). Its weight is about $400 \mathrm{~kg}$ with dimensions of $120 \times 120 \times 180 \mathrm{~cm} 3$. General view of the current mobile robot system is shown in Fig. 1.

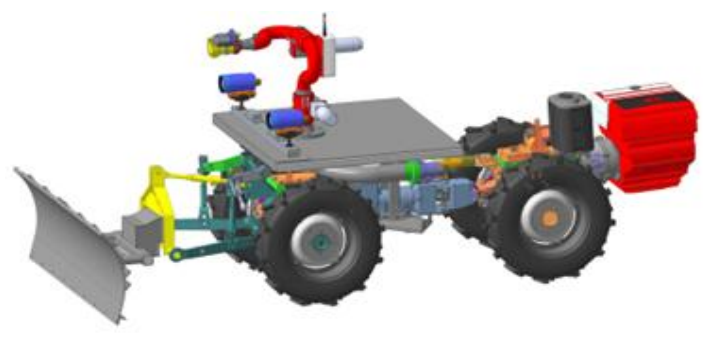

Fig 1: General view of the Belarus 132N Robotic system

\subsection{Discrete Time Model of Belarus-132N Wheeled Mobile Robot}

The robot is assumed to be moved in a flat plane where the motion $P$ can be described in $\mathrm{I}_{\mathrm{xp}}$ and $\mathrm{I}_{\mathrm{yp}}$ direction using four wheels. The direction $\theta$ determines the rotation of mobile robot in counter clockwise. The robot motion can be described in two coordinate systems: global coordinate system $\mathrm{I}_{\mathrm{x}}, \mathrm{I}_{\mathrm{y}}$ axis and Local coordinate system $\mathrm{E}_{\mathrm{x}}, \mathrm{E}_{\mathrm{y}}$ as shown in Fig. 2.

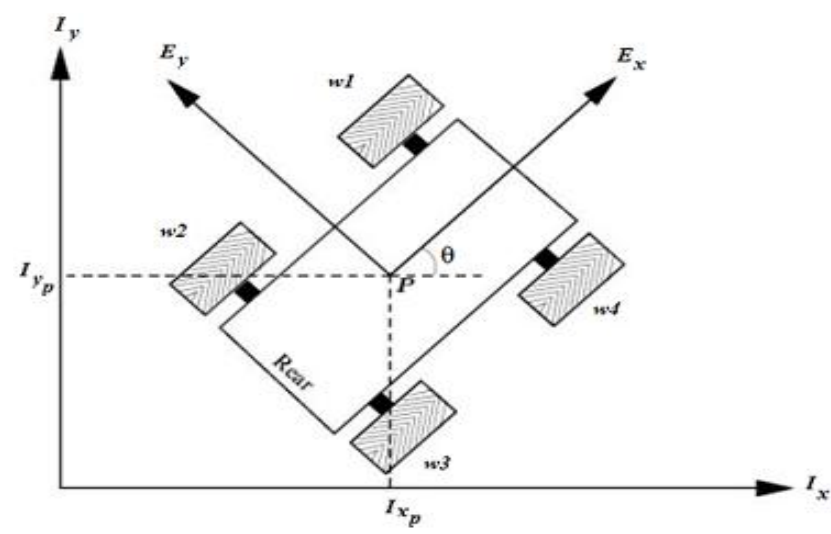

Fig 2: Location of Wheeled Mobile Robot with respect to global and local coordinate system

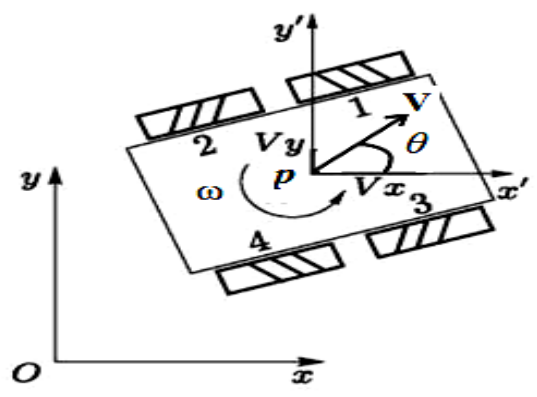

\subsubsection{Kinematic model of mobile robot}

The kinematic model [5] of a mobile robot at point $\mathrm{p}\left(\mathrm{x}_{\mathrm{p}}, \mathrm{y}_{\mathrm{p}}\right.$, $\left.\theta_{\mathrm{p}}\right)$ as shown in Fig. 3 is given by:

$$
\begin{aligned}
& \dot{x_{p}}=V \cos \theta \quad \dot{y_{p}}=V \sin \theta \quad \dot{\theta_{p}}=\omega_{p} \\
& V=-\frac{K\left(V_{1}+V_{2}\right)}{2} \quad \omega=-\frac{K\left(V_{1}-V_{2}\right)}{2}
\end{aligned}
$$

Where $\mathrm{x}$ and $\mathrm{y}$ represent the coordinates of the centre of the axis of the actuated wheels on the plane X-Y and $\theta$ is the angle formed by the longitudinal axis of the robot and the $\mathrm{X}$ axis. $\mathrm{V}$ represents the linear velocity, $\omega$ represents the angular velocity, V1 and V2 - longitudinal velocity of the wheel while $\mathrm{K}$ - drive transmission ratio. The kinematic model of a four wheeled mobile robot is shown in Fig. 4.

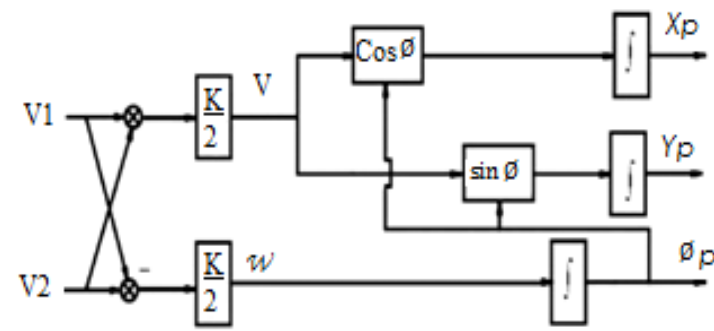

Fig 4: kinematic Model of a Wheeled Mobile Robot

\subsubsection{Discrete Time Model of mobile robot}

Considering an example break Ts and a zero-appeal hold, eq. (1) movements in discrete-time of a mobile robot at point $p\left(x_{p}\right.$ , $\left.y_{p}, \theta_{p}\right)$ to:

$$
\left\{\begin{array}{l}
x_{p}[k+1]=x_{p}[k]+V[k] \cdot \cos \theta[k] \cdot T_{s} \\
y_{p}[k+1]=y_{p}[k]+V[k] \cdot \sin \theta[k] \cdot T_{s} \\
\theta_{p}[k+1]=\theta_{p}[k]+\omega[k] \cdot T_{s}
\end{array}\right.
$$

Consequently a virtual robot, the heading $q_{d}(t)=\left[x_{d}(t) y_{d}(t)\right.$ $\left.\theta_{\mathrm{d}}(\mathrm{t})\right]$ is viewed as coming about the accompanying kinematic model, Mathematical statement of the virtual robot can be communicated as:

$\dot{x_{d}}=V_{d} \cos \theta_{d} \dot{y_{d}}=V_{d} \sin \theta_{d} \dot{\theta_{d}}=\omega_{d}$

Where $\mathrm{p}_{\mathrm{d}}=\left(\mathrm{x}_{\mathrm{d}}, \mathrm{y}_{\mathrm{d}}, \theta_{\mathrm{d}}\right)$ represents the desired pose, $V_{d}$ the desired linear speed, $\mathrm{w}_{\mathrm{d}}$ the desired angular.

Considering an example break Ts and a zero-appeal hold, eq. (4) movements in discrete-time to:

$$
\left\{\begin{array}{l}
x_{d}[k+1]=x_{d}[k]+V_{d}[k] \cdot \cos \theta_{d}[k] \cdot T_{s} \\
y_{d}[k+1]=y_{d}[k]+V_{d}[k] \cdot \sin \theta_{d}[k] \cdot T_{s} \\
\theta_{d}[k+1]=\theta_{d}[k]+\omega_{d}[k] \cdot T_{s}
\end{array}\right.
$$

Fig 3: Kinematic Position of a Wheeled Mobile Robot 


\section{OVERVIEW OF MODELPREDICTIVE CONTROL}

Model Predictive Control (MPC), is playing a vital propelled control technique for troublesome multivariable control problems and is widely implemented within industry and getting more popular to those who are interested in control to fulfill their duties. In fact predictive control would describe an approach to the control design rather than a specific algorithm. And thus an interpretation of the approach is required to define a suitable algorithm that fits the needs. If a clear and defined dynamic model is available for a process, then the model and present measurements could be utilized to reach forthcoming/future output values. At that point the proper fluctuations in the input variables can be computed in accordance of the measurements and predictions. Fundamentally, any changes in the input variables are managed with respect to the I/P-I/O relationships that are introduced by the process model. The output variables are referred as controlled variables (CVs), and on the other hand input variables are referred to as manipulated variables (MVs). In addition, the measured disturbance variables are known as (DVs) and in other terms may be called feed forward variables.

Model predictive control is well known to provide many favorable leverages; first as we begin by the process model it would start in collecting the static as well as the dynamic interaction between the input, output, and DVs; secondly, the constraints if any are existing on $\mathrm{I} / \mathrm{P}$ and $\mathrm{O} / \mathrm{P}$ which are taken into effect in the sense of systematic aspect; thirdly, the control computations are possible to interact with finding the ultimate set points, and the fourthly; building a model of accurate predication can detect, trace and flag any upcoming issues. Realizing that the main objective that MPC has been illustrated by Qin and Badgwell as: i) to avoid infringement of constraints on I/O-O/P; ii) push part or few of the $\mathrm{O} / \mathrm{P}$ variables to the set point (SP), while keeping up other $\mathrm{O} / \mathrm{Ps}$ in defined set of ranges; iii) to set a static status on the input variables; iv) and in the absence of a sensor to control the most possible process variables.

MPC is a comprehensive control system that consists of accompanying modules [6], [7], [8], [9]. MPC system block diagram is shown in Fig. 5. In order to predict the output variables values a process model is going to effectively used. Normally any variations between real and predicted outputs are going to be acting as a feedback signal to a prediction module. At every sampling instance those predictions are utilized as a part of the following MPC calculations which starts with the set point and control computations. Note that inequality constraints whether occurring on the input and output variables, can be involved in any of the above calculations.

Targets which are known as set points and serve for control calculations would be evaluated and computed from the process steady state model (linear model). When the process settings are changing the set points values change as well due to these variations in the process circumstance particularly in the changes of the inequality constraints. The changes and variations in the constraint are mainly due to fluctuations in process surroundings, apparatus, devices, etc.

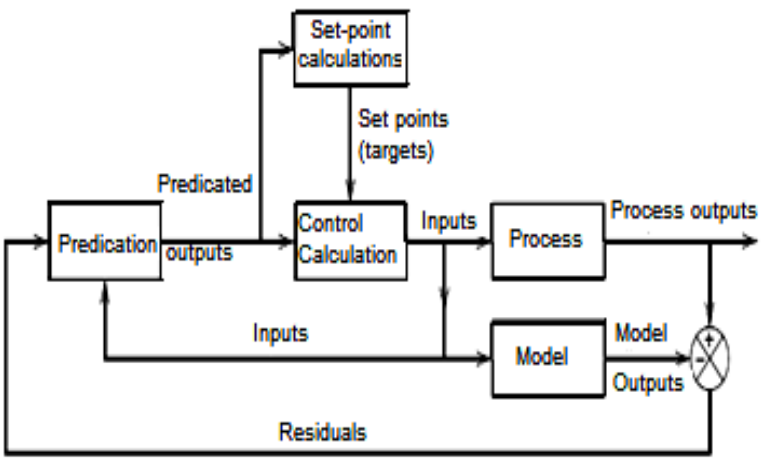

Fig 5: Block diagram for model predictive control

\section{GENERALIZED NONLINEAR PREDICTIVECONTROL}

Figure 6 demonstrates the basic principle of MPC. At a denoted time $(\mathrm{t})$ certain measurements are provided. These measurements will trigger the controller and kick it to commence predicting the forthcoming (future) dynamic act of a system over a predicted horizon called $\mathrm{Tp}$ and furthermore determines the input at which optimization of predefined open loop performance goals are met.

As long as neither disturbances exist nor mismatching model plant presence is evident, in this case if there was a way for the infinite horizon optimization issue to take place in solving it, hence at this point and at time of $t=0$ the input function can be incorporated in regards of the system in a condition where all times " $t$ " is greater or equal to zero $(t \geq 0)$. However and as a matter of fact, this is not feasible generally. Because of the factors of disturbances and the stressing mismatching model plant, the real and predicted behaviors of the system will not be the same. The integration of the feedback would depend on the acquired I/P function of the open loop and shall be ready for implementation whenever a next measurement is around.

A difference in the time among the re-calculation incident and measurement can change but regularly is expected being fixed however often it is assumed to be fixed, i.e. a measurement is going to occur throughout each $\delta$ sampling time units. During a time $(t+\delta)$ a new measurement is used, and later due to these measurements the process of both the optimization and prediction will re-run to figure out and get another recent input function (new) in conjunction with both the control/prediction horizons advancing ahead forward.

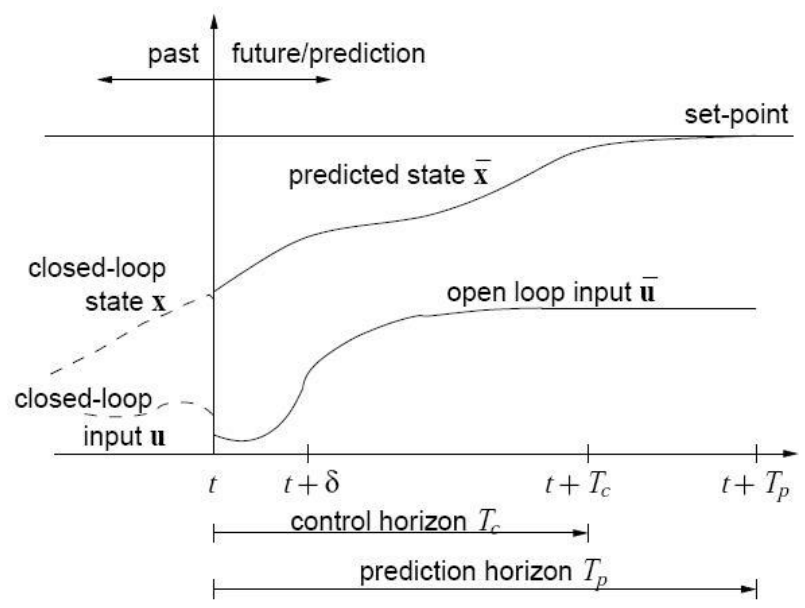

Fig 6: Principle of Model Predictive Control 
Nevertheless, the input can be assumed as constant during a sampling time $\delta$. The computations of the input that will be applied permits adding constraints on the states/inputs and more could go for optimizing certain cost function. Technically speaking the behavior of predicted system is going to be different from a closed loop; therefore the stability of the closed loop will require more analysis to achieve it.

According to the literature a nonlinear set of differential equations as outlined and shown below will touch base the issue of stabilization as we move through a set of systems [6], [7]:

$\dot{X}(t)=f(x(t), u(t)), \quad X(0)=x_{0}$,

Which is represented to input and state constraints as set out below,

$U(t) \in \mu \forall t \geq 0 \quad x(t) \in X, \mu \forall t \geq 0$

Where $\mathrm{x}(\mathrm{t}) \in X \subseteq \mathbb{R}^{n}$ and $u(t) \in \mu \subseteq \mathbb{R}^{m}$ denotes the states vectors along with the inputs. The set of feasible input values is denoted by $\mathrm{X}$. Note that $\mu$ and $\mathrm{X}$ shown as constraints according to the below form:

$\mu:=\left\{u \in \mathbb{R}^{m} \mid \operatorname{Umin} \leq u \leq \operatorname{Umax}\right\}$,

$X:=\left\{x \in \mathbb{R}^{n} \mid X \min \leq x \leq X \max \right\}$,

Note that $\mathrm{u}_{\min }, \mathrm{u}_{\max }$ as well as $\mathrm{x}_{\min }, \mathrm{x}_{\max }$ are assumed as constant vectors.We presume that the aim and main objective of a control object (6) is to assure:

$$
\begin{aligned}
& \lim _{t \rightarrow \infty}\left\|x(t)-r_{x}(t)\right\|=0 ; \\
& \lim _{t \rightarrow \infty}\left\|u(t)-r_{u}(t)\right\|=0 ;
\end{aligned}
$$

Where the vector functions; $r_{x}(t)$ and $r_{u}(t)$ identify a preferred passage of object.

The control of objects' moves can be set or regulated by a specific functional; (1).

$$
J_{0=} J_{0}(x(t), u(t))
$$

Optimal control problem would definitely need to look for a control action of a specified class, which assure the accomplishment of setup goals (9) without forgetting the constraints $x(t) \in X$ and $\forall t \in[0, \infty)$ andhence the functional minimizes (10).

To overcome the problems of optimal control various techniques are used and applied in the multiple forms and approaches where we can take as a sample the theoretical applications in model predictive control that was considered as the backbone of feedback based on the structure of control action that rely on and utilize the measured information on the object's state.

To illustrate and go through a predictive control theory, we will need to consider a mathematical model of the control object (6) as well as we will need a set of differential equations that can be such as:

$\dot{\bar{X}}_{i}(\tau)=\left.\bar{f}(\tau, \bar{x}(\tau), \bar{u}(\tau)) \bar{x}\right|_{\tau=t} \cdot \dot{\cdot}=x(t)$,
Additionally, assume that the function $\bar{f}$ is set for any permissible control $\bar{u}(\tau) \equiv u(\tau)$ vector functions $x(\tau)$ and $\bar{x}(\tau)$, meets the system expectations (6) and (11) respectively; and being nearby at a pace of $\tau \in[t, \infty)$.

The identified systematic model of differential equations (11) has formed a close associative relation between the predictive and mathematical models (6) of the control object.

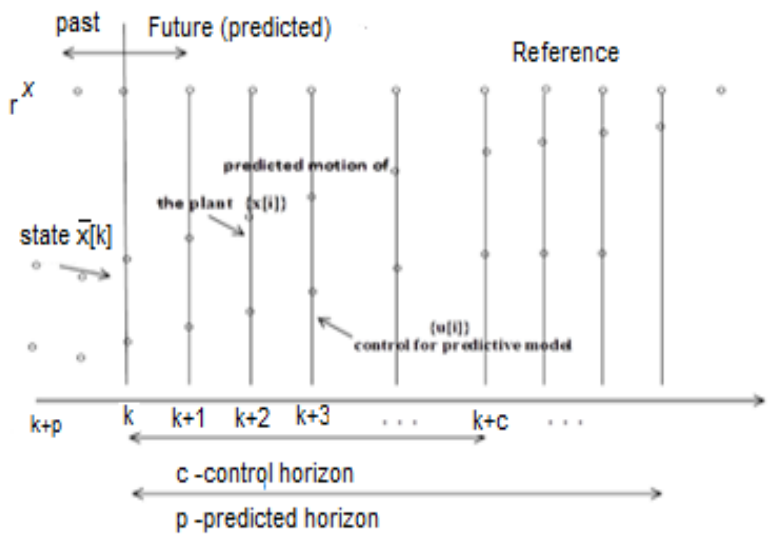

Fig 7: Predictive plant movement

A delayed time $\boldsymbol{\tau}$, at the $\mathrm{x}$-axis would be and during an initial state, let us consider $\tau=\mathrm{T}$. So far, the control object with an unidentified precise model of the form (6) is forward moving through the influence of $u(\tau)$, certain control executed feedback scheme, and through the time it was in a state.

Let us consider an explicit control that would work as a function of time interval and being having a full integration with the system (11) at a defined interval with the initial state. The resultant incomplete solution will be viewed as the predicted behavior of the control object however through the zone of prediction horizon as shown in Fig. 7.

We need to stress out that because of the differences that falls among the dynamics of a real object and a predictive model of transit on the sector. Such a segment will be assumed an entire difference, knowing that any sort of concurrence is valid at the initial point only.

At this point we can formulate a mathematical problem of choosing the optimal control based on the forecast. Further we will suppose that the purpose of management is to provide a predetermined pattern of behavior (11), it is determined as in (9), and vector functions $r_{x}(t)$ and $r_{u}(t)$, where $X \in E^{n}$ - the state vector; $u \in E^{m}$ - the vector control, $t \in[0, \infty)$.

The structure of all controllers of the MPC family follows a strategy as shown in Fig. 8 as follows: (i) Future output values are calculated every time $t$; (ii) Future control signals using an optimization method, process, and the reference trajectory is calculated so as to converge as much as possible; (iii) Typically, this optimization method is quadratic as a function of the difference between the predicted output and the predicted trajectory; (iv) The control signals measured only the first sample found. 


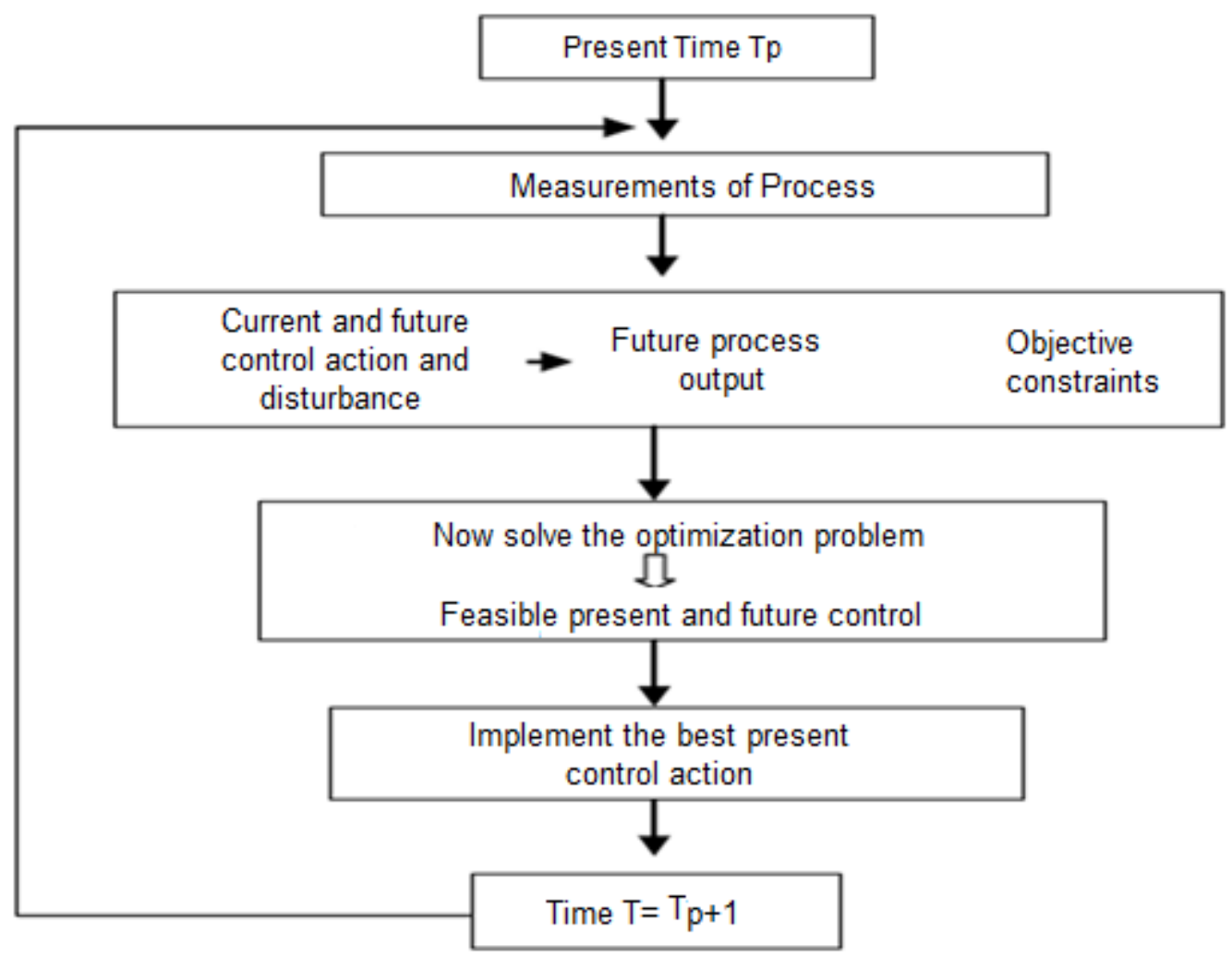

Fig 8: Model Predictive Control Scheme

By introducing model predictive control approach a formulation of a constrained optimal control algorithm is and incorporated throughout the receding horizon scheme [10].

It is obvious that any sort of a model is constructed as a result of the physical plant characteristics as well as the control problem normally and initially would be formulated by taking into account the objectives and constraints of the system.

\subsection{Fuzzy Modeling}

A highly nonlinear system controlled by a linear Model Predictive Controller may not give a satisfactory control solution. To solve this problem, Nonlinear Model Predictive Control methods are proposed. But it is not easy to apply these methods practically since the nonlinearity increases the computational effort. Nonlinear process is defined by a set of linear sub-models and algorithms decreasing the computational effort to design controllers are proposed. In this study, an alternative approach to these methods, Fuzzy Model Predictive Control approach is proposed.

According to the Fuzzy Model Predictive Control approach, a nonlinear process is described by a fuzzy convolution model that consists of a number of linear or quasi-linear subsystems. For each subsystem, a Model Predictive Controller is designed and the control effort is fuzzily merged by using the same rules used to merge subsystems.

Fuzzy logic, linguistic variables are expressed based on an expert knowledge, it is a method used in order to quantify the value of linguistic and linguistic rules [11]. Fuzzy logic is frequently used in the modeling of complex systems. Depending on the nature of inference rules, there are three different models of fuzzy systems: (i) Linguistic fuzzy models; (ii) Fuzzy related models; (iii) Takagi - Sugeno (TS) fuzzy model [12].

The first two categories above are known as Mamdani fuzzy models. Accordingly, TS fuzzy modeling structure can be defined as follows: (i) The input space separated into sub spaces; (ii) Then each sub-space system model converges to more simple linear models; (iii) Finally, as required by fuzzy subsystem models of the rule base on the weighted average, the overall structure is created fuzzy model [4].

The path above in reference [10] Fuzzy Bend (Convolution) is expressed as the model. Accordingly, a non-linear system is decomposed into $p$ sub system and the behavior of each subsystem to be linear or linear-like. TSA's modeling requirement, a fuzzy linear-like pattern must be defined for each sub-claims [13]. These definitions are fuzzy inference (fuzzy implication) is called and in the sub-region $\left(\mathrm{R}^{\mathrm{i}}, \mathrm{i}=1,2\right.$, $. ., p)$ is defined. In this way, the cause and effect relationship model, the control signal $\mathbf{u}$, output $\mathbf{y}$, and it is represented by discrete time difference equation in terms of the sampling instant $\mathbf{n}$. Each region is represented by a fuzzy Cartesian product as follows [10].

$\mathrm{R}^{\mathrm{i}}=\mathrm{y}(\mathrm{n}) \times \mathrm{y}(\mathrm{n}-) 1 \times \ldots \times \mathrm{y}(\mathrm{n}-\mathrm{m}+) 1 \times \mathrm{u}(\mathrm{n}) \times$

$\mathrm{u}(\mathrm{n}-1) \times \ldots \times \mathrm{u}(\mathrm{n}-1+1)$

$\boldsymbol{y}(\boldsymbol{n}-\boldsymbol{j})$ in the above expression, $\boldsymbol{n}-\boldsymbol{j}$ instantly measured output value $\boldsymbol{u}(\boldsymbol{n}-\boldsymbol{j}), \boldsymbol{n}-\boldsymbol{j}$ is instantly measured input values.

Fuzzy inference rules are consisting of symbolic premise of "if" part consisting of the numerical results and the phrase "then" consists of. In other words, the lower bending fuzzy model has a structure as follows [10]:

$\mathrm{R}^{\mathrm{i}}$ : if 
$y(n) A_{0}^{i}, y(n-1) A_{1}^{i}, \ldots ., y(n-m+1) A_{m-1}^{i}$

and

$\mathrm{u}(\mathrm{n}) \mathrm{B}_{0}^{\mathrm{i}}, \mathrm{u}(\mathrm{n}-1) \mathrm{B}_{1}^{\mathrm{i}}, \ldots ., \mathrm{u}(\mathrm{n}-1+1) \mathrm{B}_{1-1}^{\mathrm{i}}$

Then;

In the above-mentioned common:

- $\boldsymbol{A} \boldsymbol{i}_{j}$, i'th fuzzy inference Amedaki y (n-j) of the output fuzzy sets.

- $\boldsymbol{B} \boldsymbol{i}_{j}$, i’th fuzzy inference Amedaki (n-j) of the input fuzzy sets.

- $\boldsymbol{h} \boldsymbol{i}_{j}$,i'th fuzzy inference Amedaki impulse response coefficients are.

- $\mathrm{T}$ is the model horizon.

- $\Delta(\mathrm{n}), \mathrm{u}(\mathrm{n})$ and $\mathrm{u}(\mathrm{n}-1)$ is the difference between [10].

In this study, instead of impulse response of the subsystem, differential equations were used. This change did not cause any difference in the rule base, just brought out a calculation of the difference in the form of sub-systems.

All pieces are pin fuzzy inference in the fuzzy bending model. System output y $(n+1)$, the weighted average of the output value calculated by the fuzzy inferences can be found by [10].

$y(n+1)=\frac{\sum_{j=1}^{p} w^{j} y^{j}(n+1)}{\sum_{j=1}^{p} w^{j}}$

$\mathrm{W}^{\mathrm{j}}$ term in the above equation, $\mathrm{j}$ th fuzzy inference degree of reality, that is the multiplier ignition. Ignition factor fuzzy sets "if" part is calculated on the basis of the following form [10]:

$w=\bigwedge_{i} A_{i}^{j} \wedge_{k} B_{k}^{j}$

We use Triangular membership functions for each fuzzy linguistic value as shown in Fig. 9 of the total equal to 1.

$$
\sum_{j=1}^{p} w^{j}=1
$$

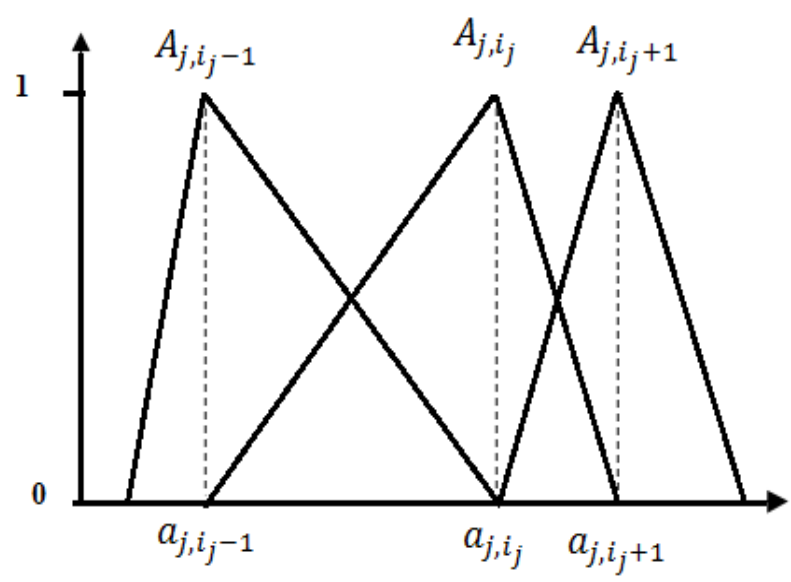

Fig 9: Membership functions used for the fuzzy model
Accordingly, $\mathrm{y}(\mathrm{n}+1)$ is transformed to equation with the following form [13]:

$y(n+1)=\sum_{j=1}^{p} w^{j} y^{j}(n+1)$

Equation (13) for each fuzzy sub-models as seen when calculating $\mathrm{y}^{\mathrm{j}}(\mathrm{n}+1)$ and $\mathrm{y}^{\mathrm{j}}(\mathrm{n})$ is used instead of using $\mathrm{y}(\mathrm{n}) \cdot n$ is immediately used such an expression in order to minimize the estimation error is known $\mathrm{y}(\mathrm{n})$ [10].

After the output values calculated FMPC structure will be introduced. However, it is necessary to explain the first classic MPC structure to explain the first classic MPC structure for it.

\section{PROPOSED SYSTEM}

Differential GPS navigation system which complements the fixed network of terrestrial base stations, improves position accuracy of one meter. But this accuracy is not quite enough to ensure the normal operation of the control systems of vehicles, robots and unmanned aerial vehicles.

A new method to obtain accuracy is a combination of work standard GPS, RVS (Robot Vision System), and QVS (Quadcopter Vision System)as shown in Fig.10. The GPS system provides in this case, the exact locations of the binding data, RVS, and QVS are used to ensure the continuity of location determination process. Combining the data from the GPS, RVS, and QVS allow obtaining the exact value of the coordinates.

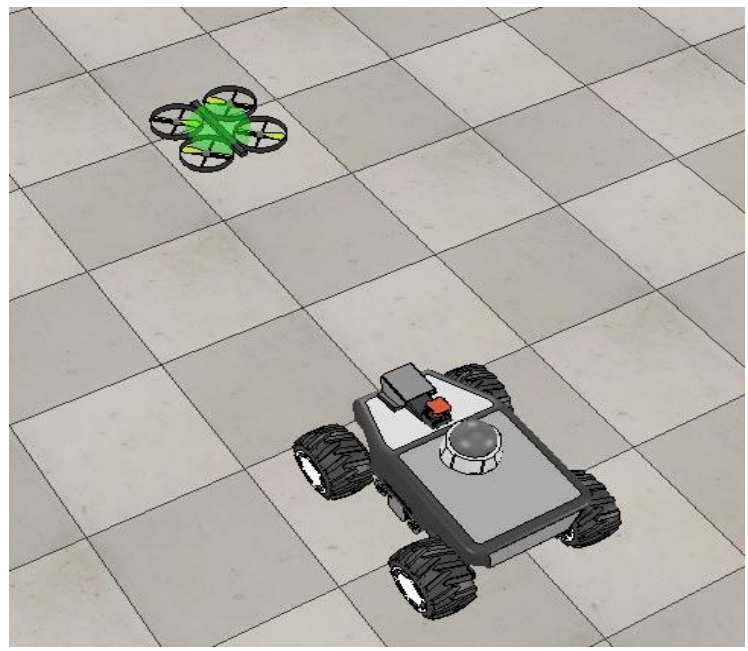

Fig 10: General scheme of QMRS 


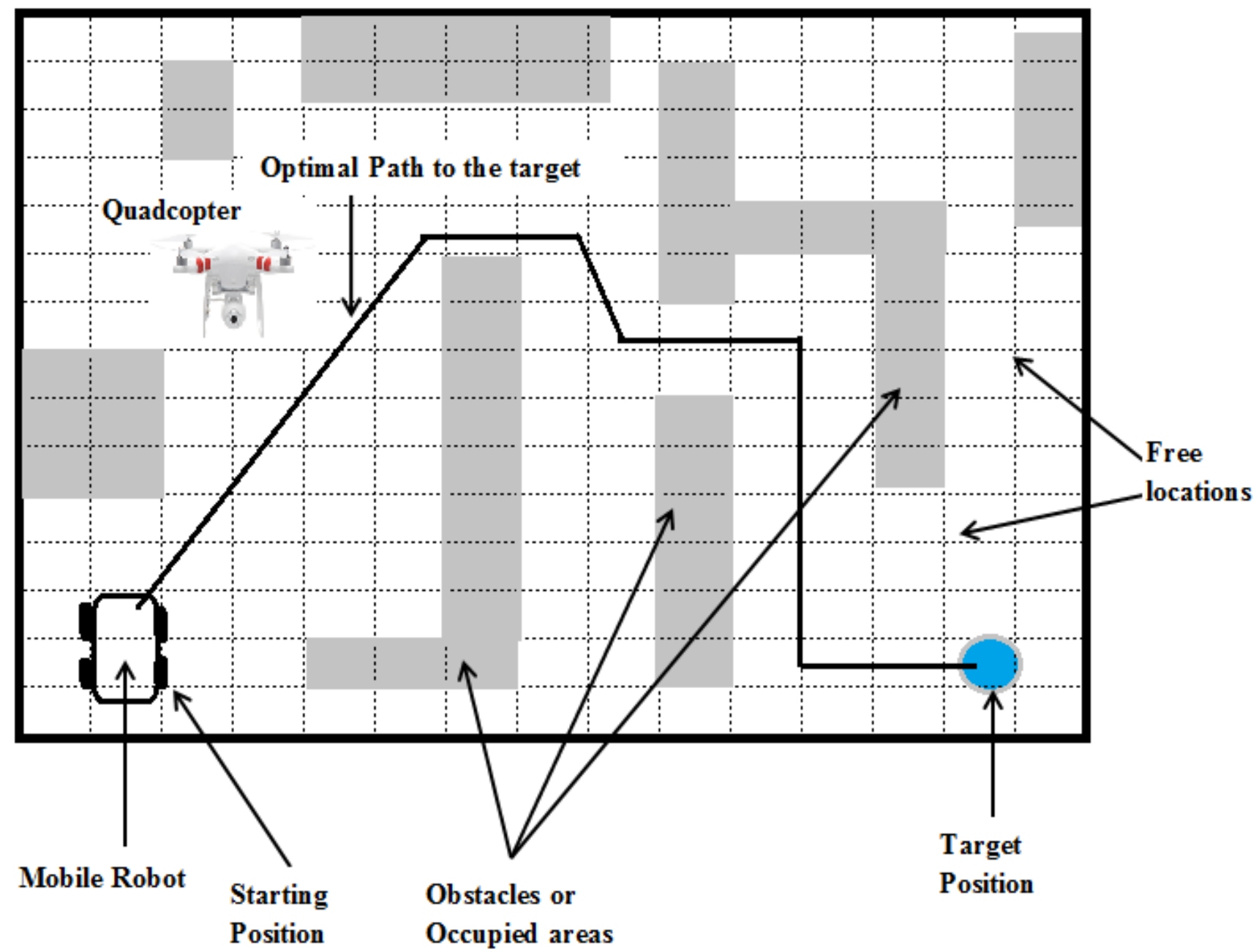

Fig 11: Finding the Optimal Path of Mobile Robot

Using this method allows us to achieve a very high level of accuracy, but requires computing resources, which do not have the most powerful microprocessors and microcontrollers. This will create a highly specialized navigation systems (Fuzzy Logic) designed for each specific area, for car-robots, agricultural robots and other automated equipment for civilian and military purposes. Moreover, highprecision positioning modules may become available in smart-phones and other portable electronics without increasing their ultimate cost.

\subsection{Detection and Object tracking}

Automatic detection and tracking of objects observed in a sequence of images is an actual problem, often occurring in the development of navigation systems manned and unmanned aircraft. At the same time one of the most important challenges is to ensure detection and tracking under uncertainty relative spatial arrangement of the camera and lens. There detection and tracking task airfield on board the aircraft in conditions when the initial relative positioning of the airfield and the aircraft is unknown. An essential requirement for the developed algorithm is the ability to implement it in the onboard data processing systems.

\subsubsection{Robot Vision System (RVS)}

RVS use image processing system to extract elements or objects by processing each image in the video sequence. RVS projects each found object with a vertex on a RVS-smartmap-graph. Each found object has the following properties:

a) Identifier - a unique number assigned to the new object detection. Number assigned to this object as long as there is no decision on the loss of the object;

b) Coordinate and orientation of object $\left(\mathrm{x}_{\mathrm{i}}, \mathrm{y}_{\mathrm{i}}, \theta_{\mathrm{i}}\right)$;

c) Width of new object;

d) Length of new object;

e) Height of new object;

f) Life Time - the number of frames of a video sequence in which the object was present since the first detection;

g) Object type.

\subsubsection{Quad-copter Vision System (QVS)}

QVS use image-down-view processing system to extract elements or objects by processing each image in the video sequence. QVS projects would each found an object with a vertex on a QVS-smart-map-graph. Each found object has the following properties:

a) Identifier - a unique number assigned to the new object detection. Number assigned to this object as long as there is no decision on the loss of the object; 
b) Coordinate and orientation of object $\left(\mathrm{x}_{\mathrm{r}}, \mathrm{y}_{\mathrm{r}}, \theta_{\mathrm{r}}\right)$;

c) Width of new object;

d) Length of new object;

e) Height of new object;

f) Life Time - the number of frames of a video sequence in which the object was present since the first detection;

\section{g) Object type.}

\subsubsection{Trajectory Analysis of RQVS}

Trajectory analysis algorithm is based on the decomposition of a smart-map-graph. At the entrance of the algorithm are the measurement data - parameters of an image extracted by processing each image in the video sequence. Downstream - a list of found objects and their properties, in which each element of the list is marked with a unique number.

The first step of the algorithm is to construct a smart-mapgraph; the first group of vertices is the set of objects known on the current frame, and the second - the set of connected regions found on the frame. The algorithm will measure the distance between each pairs of vertices located at Smart-MapGraph.

The algorithm use information of object identifier from both RVS and QVS, if the objects appear twice (coincide) on smart-graph-map i.e. the object has been detected by both systems so the algorithm neglect one of them.

A recursive procedure is repeated for each received subgraph. The feasibility of using this algorithm is caused by the presence of such parameter of the detected object, as the time of life. At the stage of preliminary detection of the desired object is taken with the greatest lifetime and thus reduces the probability of false detection.

With the trajectory analysis algorithm analyzes a list of objects found in the previous step. The result of the algorithm for tracking the object coordinates of the object are taken, having previously memorized identifier. The coordinates of the found object can then be used to adjust the rate of moving of Mobile Robotas shown in Fig. 11.

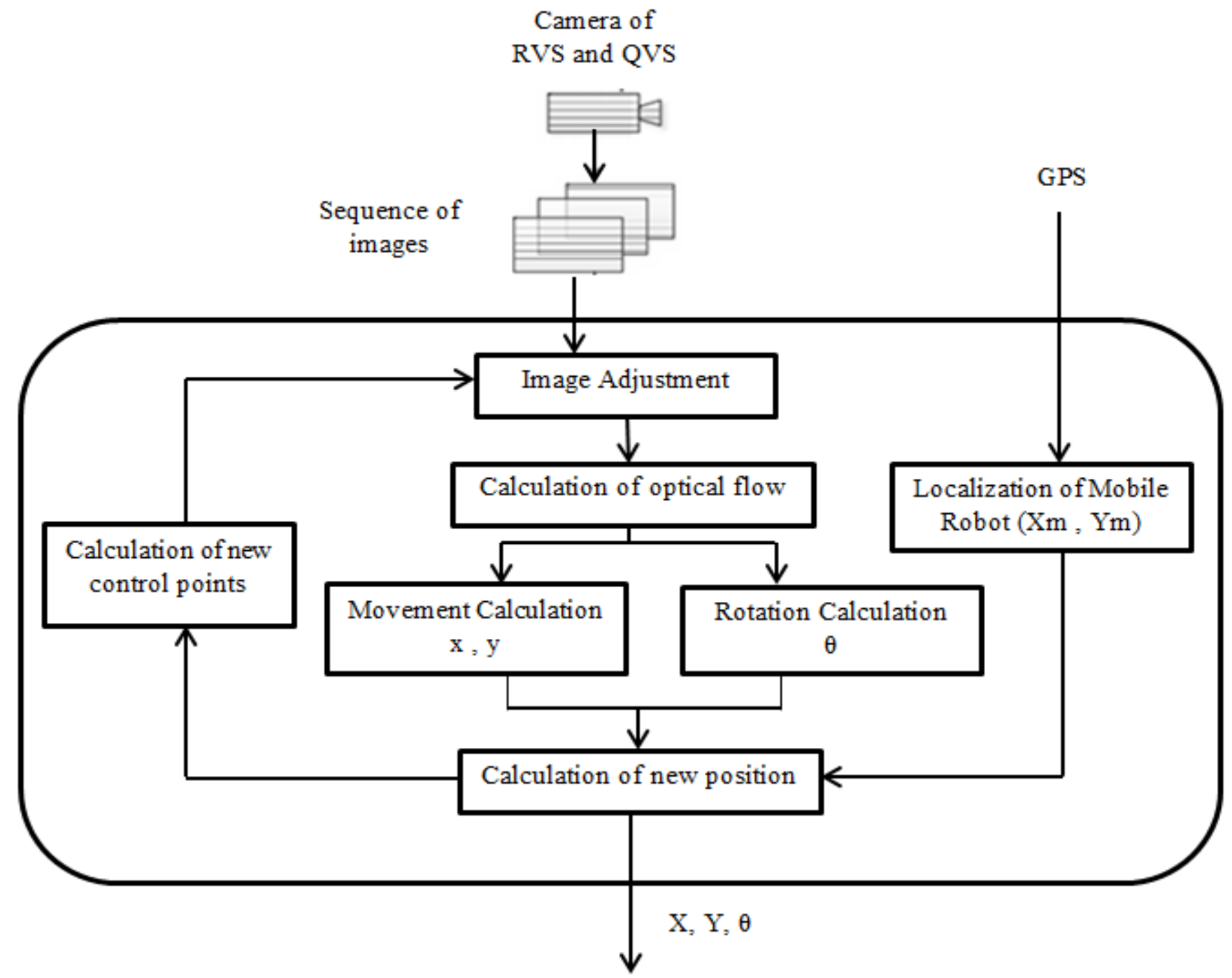

Fig 12: General scheme of the proposed system of FMPC 


\subsection{Calculation of new position $(x, y, \theta)$}

Fuzzy Model Predictive Control (FMPC) process input variables from different resources such as: GPS, robot vision system (RVS), and quad-copter vision system (QVS) in order to achieve localization i.e. Cartesian coordinates $(\mathrm{X}, \mathrm{Y})$ and the orientation $\theta$.

The algorithm of Fuzzy Model Predictive Control can be described as follows.

1) Use the coordinate of GPS for Mobile robot.

2) Calculate the optical flow for the frame for both RVS and QVS of smart-graph-map.

3) The total optical flow into two parts: the "terrestrial" and "un-terrestrial ".

4) In the "un-terrestrial " of the move to a cylindrical coordinate system and calculate the angle of rotation $\theta$ with respect to the last two frames, thereby determining the angle of rotation.

5) The "terrestrial" side of the highlight optical flow vectors and to calculate displacement in the plane XOY, received vector $(\mathrm{x}, \mathrm{y})$.

6) Add ( $\mathrm{x}, \mathrm{y})$ to the original position of the mobile robot $(\mathrm{Xm}, \mathrm{Ym})$ in order to get a new position of $(\mathrm{X}, \mathrm{Y})$.

7) Go to step 1 for the next frame. Periodically update the key points. In general, the process of calculating the optical flow is shown in Fig.12.

\section{CONCLUSION AND FUTURE WORK}

In this paper, the motion control system and algorithm for a Mobile Robot was presented. The robot positioning and localization was calculated using Fuzzy Model Predictive Control.

The algorithm of FMPC use data from different inputs such as: GPS, RVS, and QVS. The program is able to take into consideration the occurrence of obstacles along the path of travel. The successful generation and simulation of two different kinds of static obstacles from RVS and QVS are projected on Smart-Graph-Map.

The program has been developed in such a way that it increases efficiency of moving and localization of mobile robot by the help of quad-copter which can be introduced into other related applications for unmanned navigation designs.

\section{REFERENCES}

[1] D. Clarke, C. Mohtadi, and P. Tuffs, Generalised
Predictive Control-Part I. The Basic Algorithm, Automatica, Vol. 23, No. 2, pages 137-148, 1987.

[2] J. B. Rawlings, Tutorial Overview of Model Predictive Control, IEEE Control Systems Magazine, Vol. 20, No. 3, June 2000, pages 38-52.

[3] A. Jadbabaie, J. Yu, and J. Hauser, "Stabilizing receding horizon control of nonlinear systems: A control Lyapunov function approach," in Proceedings of the American Control Conference, San Diego, CA, USA, 1999, pp. 1535-1539.

[4] G. DE Nicolao, L. Magni, and R. Scattolini, "Stabilizing receding horizon control of nonlinear time-varying systems," IEEE Transactions on Automatic Control, vol. 43, no. 7, pp. 1030-1036, 1998.

[5] R. Siegwart, I. R. Nourbakhsh and D. Scaramuzza," Introduction to Autonomous Mobile Robots ",2nd Edition,2004, ISBN: 978-0-262-01535-6.

[6] I. A. Elzein, Y. N. Petrenko, "An Integration of A Predictive Control Model and MPPT for PV Station". International Conference on Smart Systems and Technologies, Osijek, Croatia. IEEE Trans. ISBN:978-15090-3718-6. pp. 275-280, 2016.

[7] Sotnikova MV. Development toolkit for solving control problems with non-linear predictive models in MATLAB Tr. V Int. Conf. "Design of engineering and scientific applications in the environment MATLAB", 2011, p. 299-319.

[8] Veremey EI, MV Sotnikova, Plasma stabilization on the basis of the forecast of a steady linear approximation, St Petersburg. Univ. Ser. 10. 2011, Vol. 1, p. 116-133.

[9] Sotnikova MV, Questions of stability of motion in control systems with predictive models, Bulletin of Voronezh State Technical University, 2012, p. 72-79.

[10] Huang, Y. L. and Lou, H. H. and Gong, J. P. and Edgar, T. F., 2000. Fuzzy model predictive control, IEE Transactions on Fuzzy Systems, 8(6).

[11] Passino, K. M. and Yurkovich, S., 1998. Fuzzy Control, Addison-Wesley Longman, California, 52.

[12] Nikolaou, M., Model Predictive Controllers: "A Critical Synthesis of Theory and Industrial Needs", Houston, TX 77204-4792.

[13] Takagi, T. and Sugeno, M., 1985. Fuzzy identification of systems and its application to modeling and control, IEEE Trans. Syst., Man, Cybern.,15, 116-132. 